\title{
Physical activity from early childhood to adolescence: a literature review of issues and interventions in disadvantaged populations
}

\author{
Murray JN Drummond \\ School of Education, Flinders University
}

Claire E Drummond

Social Health Science, Flinders University

\section{Jim Dollman}

School of Health Sciences, University of South Australia

\author{
Liz Abery \\ Social Health Science, Flinders University
}

\begin{abstract}
Background: This paper is based on a report commissioned by the South Australian Health Department to undertake a literature review identifying key physical activity interventions in 'different' populations. This paper presents the findings from the literature surrounding youth from early childhood through to adolescence only.

Methods: We conducted a comprehensive literature search using the following online academic databases: Proquest, Informit, Blackwell Synergy, Sage Publications, CINAHL and Cochrane. The search targeted peerreviewed articles, systematic reviews and evaluations. Each search used the term 'physical activity and', using the Boolean 'or' exercise. Using the Boolean 'and' combinations of the following words were added: low socioeconomic position 'or' low socioeconomic status, culture, intervention,
\end{abstract}


health promotion, evaluation, strategies, South Australia, Australia. We also reviewed Australian government websites.

Results: There are myriad reasons for the success or failure of physical activity interventions for youth ranging from parental influences, gender and age, culture, socioeconomic status, and social and physical environments.

Conclusions: Multi-faceted approaches to interventions are required to achieve optimal outcomes for youth from early childhood through to adolescence. School provides an ideal site to engage students in physical activity. However 'curriculum-only' strategies do not work. Engaging parents in the interventions will heighten the probability of success.

\section{Introduction}

International epidemiological evidence from the past two decades has consistently identified physical activity as a major modifiable risk factor in the reduction of mortality and morbidity from many chronic, non-communicable and potentially preventable diseases (Bauman, Owen and Leslie 2000; Stephenson et al. 2000; World Health Organization 2004). Such diseases place a huge economic burden on health services and inflict considerable costs on society (World Health Organization 2004). In Australia it is estimated physical inactivity is responsible for approximately 8000 deaths per year (Mathers, Vos and Stevenson 1999) and the impacts on the direct costs of health care are estimated at in excess of $\$ 400$ million and rising, indicating a significant burden of disease as well as substantial economic burden to the Australian population (Stephenson et al. 2000). If indirect costs such as lost employment time and social impacts were also considered this figure would be much greater (Bauman et al. 2002). Evidence suggests that physical inactivity ranks second behind tobacco in contributing to mortality and morbidity in Australia and in turn the need for control to prevent disease (Mathers et al. 1999; Stephenson et al. 2000). This raises concern about the connection between inactivity and health risk while highlighting the importance of physical activity as a public health priority for health promotion and disease prevention.

In Australia, there is universal interest in increasing levels of participation in physical activity at a population level (Egger et al. 1999; World Health Organization 2004). Socioeconomic circumstances and disadvantage shape and constrain available options to participate in physical activity (Short 2004). Of all of the identified areas of health risk associated with physical inactivity, those within low socioeconomic and disadvantaged groups are more likely to be affected (Egger et al. 1999; Jury et al. 2006). Developing interventions and strategies that target groups at greater risk is vital. Identifying environments and determinants that have a positive or inhibiting impact on physical activity outcomes is important (Burton et al. 2007). The challenge for policy makers is to create and maintain effective practice based on evidence to ensure that best-practice interventions are implemented in an effort to encourage an increase in activity for all ages and circumstances (Bauman et al. 2000). In addition, physical activity trends must be continually monitored, and interventions in place evaluated to assess effectiveness to determine future direction 
that responds to appropriate need (Bauman, Ford and Armstrong 2001; Bauman et al. 2002).

The initial aim of this commissioned research for the South Australian Department of Health (Drummond, Drummond, Dollman and Abery 2008) was to provide a definition of physical activity, identify physical activity trends of Australian children and adults, and outline potential approaches to physical activity interventions. More specifically the research was designed to investigate physical activity interventions in 'different' populations. In particular, low socioeconomic and disadvantaged groups were investigated as they represent an area where limited knowledge is available and specific interventions are rare. Childhood and adolescence has been identified as a key area of focus in this paper as these areas were noted in the literature as being pivotal to long-term health and an area in which physical activity interventions require clarity.

In this paper we will provide a thematic presentation of the findings from the extensive literature review on physical activity and physical activity interventions among children and adolescents from low socioeconomic and disadvantaged populations. Our recommendations will inform future intervention.

\section{Methods}

We searched the following databases, targeting peer-reviewed articles, systematic reviews and evaluations between January and July 2008: Proquest, Informit, Blackwell Synergy, Sage Publications, CINAHL and Cochrane. We also viewed Australian government websites to access statistics, policy guidelines and initiatives targeting physical activity. Using the Boolean method, 'and' combinations of the following words were added: low socioeconomic position 'or' low socioeconomic status, culture, intervention, health promotion, evaluation, strategies, South Australia, Australia. Inclusion criteria were as follows: low socioeconomic and disadvantaged groups; physical activity in conjunction with other lifestyle issues such as nutrition and obesity; physical activity a primary focus or where physical activity was measured independently of other factors; physical activity patterns and trends within South Australia and Australia; physical activity interventions; and literature published in the last 10 years. We sought a global picture when investigating interventions and we considered papers on countries with a similar culture and socioeconomic status to Australia and published in English. Each paper was independently screened by two people to determine if it met the inclusion criteria (Salmon et al. 2007). We subsequently conducted manual searches of the reference lists of suitable retrieved articles. This approach proved most beneficial in identifying key researchers and interventions in the areas of interest and as a result the literature search proved to be an ongoing process.

\section{Results and discussion}

Within the larger review, we used 114 articles. We explored influences on physical activity throughout the lifespan within the context of socioeconomic status, culture and disadvantage. For the purpose of this paper, we have identified themes and we discuss the correlation between physical activity, socioeconomic status and culture 
in relation to youth from early childhood through to adolescence only. We highlighted interventions that encourage physical activity among these groups. We have included subthemes such as environment, settings and policy where appropriate.

\section{Physical activity of infants and pre-school children}

The review found no specific Australian recommendations for minimum activity levels for pre-school children (i.e. under 5 years of age). However, it is suggested that opportunity for movement is provided and encouraged to develop sensory and motor skills (Department of Health and Ageing 2004a). Early childhood is a time to promote behaviours that follow through childhood (Campbell and Hesketh 2007). While there is limited evidence around physical activity trends in infancy and early childhood, research indicates that physical activity levels are likely to decline throughout childhood (Salmon et al. 2005). Sedentary behaviour is an area of concern. American data (Campbell and Hesketh 2007) indicates that 17 per cent of 0-11 month and 48 per cent of 12-23 month old children in the US watch more than two hours of television daily with the proportions increasing as children become older. Children of this age are unlikely to turn on the television themselves, therefore educating parents about the benefits of decreased viewing time and replacing it with activity is vital. Kahn et al. (2002) concur, claiming that family is the primary behavioural influence on young children. The home environment is pivotal with young children as they are not usually in control of decisions regarding behaviour (Ferreira et al. 2006).

\section{Interventions}

While pre-school children have been included along with other age groups in some studies of physical activity interventions, we only found one recent review relating specifically to children five and under. Campbell and Hesketh (2007) conducted a systematic literature review investigating dietary and physical activity patterns and sedentary behaviours among zero to five year olds. Of the nine studies reviewed citing evidence for this age group, five incorporated the promotion of physical activity and/or reduction in television viewing. All of these studies were American. The studies were conducted in a variety of settings including home, groups, primary care and childcare. Many targeted low socioeconomic groups or at-risk families, with one study focusing on highly educated and employed families. The design of the studies varied yet all used multiple strategies incorporating education, support and literature (Campbell and Hesketh 2007). All showed some effectiveness in increasing physical activity and reducing television viewing of not only the children but also the parents. Changes in the behaviour of the children were influenced by improved parenting skills and competencies gained through education, support and repetition of the messages.

\section{Summary}

Early childhood provides an ideal opportunity to develop lifestyle behaviour strategies that potentially carry on throughout childhood (Campbell and Hesketh 2007), although this cohort is understudied. Enhancing parental skills not only 
benefits the child but also the parent. Due to the paucity of evaluated interventions in this age group it is an area that warrants further investigation.

\section{Children and adolescents}

Being physically active may be assumed as a primary characteristic of childhood (Raudsepp 2006). Australian guidelines recommend children and adolescents partake in 60 minutes to several hours of moderate to vigorous physical activity daily (Department of Health and Ageing 2004a, 2004b). Variation in research methods, definition of what constitutes physical activity and lack of baseline data make it difficult to determine changes in physical activity trends accurately (Dollman and Pilgram 2005). Despite mixed evidence as to whether some physical activity levels have declined (Dollman and Pilgram 2005; Salmon et al. 2005) or increased (Okely et al. 2008) in Australian children and adolescents over time, current trend data suggest inactivity levels are still significant (Australian Bureau of Statistics 2006; Spinks et al. 2006; Trost 2005). In addition there is concern about the use of electronic media during the leisure time of children and youth. Children who spend more than the recommended two hours a day using electronic media are most likely not to meet physical activity recommendations (Spinks et al. 2006). As Spinks et al. (2006) have suggested, one potentially replaces the other. Although, in contrast, Harrison et al. (2006) found that an intervention that was unsuccessful in reducing television viewing was still able to increase physical activity patterns in a study of Irish primary school children. This indicated that screen-based activities may not necessarily replace physical activity.

Participation in physical activity by children and adolescents is affected by many factors. The following is a thematic analysis of these factors found throughout the literature.

\section{Parental support}

Parents are fundamental to organising, funding and supporting involvement in physical activity for children and adolescents (Raudsepp 2006). Potential difficulty arises when, due to family structure, neither parent is able to share leisure activities with their children (Martin et al. 2005). Social and financial support may vary between single and two-parent families (Raudsepp 2006). Time constraints further impact on the opportunity to encourage children and adolescents to participate in physical activity where work commitments encroach on available leisure time, particularly in single-parent families (Martin et al. 2005). Brunton et al. (2005) found that support and encouragement from parents facilitated physical activity in children and also the effectiveness of interventions (Ferreira et al. 2006).

\section{Parental involvement in physical activity}

Past research has suggested that parents' activity patterns are reflected in the levels of children's activity (Fogelholm et al. 1999). However, research conducted by Martin et al. (2005) and Ferreira et al. (2006) identified a shift in this view with parental involvement in physical activity no longer being influential in the uptake of physical activity in children. This could be due to changes in family dynamics in 
recent years. Where there is less shared family time for physical activity the role of parent modelling is diminished.

\section{Gender and age}

Recent South Australian data support the premise that boys under 15 years of age are more likely than girls to participate in physical activity (Salmon, Telford and Crawford 2004; Trost 2005) but are also more likely to spend more than the recommended two hours per day on screen-based activities (Jury et al. 2006; Salmon et al. 2004). Childhood represents a critical stage of development and therefore a vital time to influence attitudes and behaviour (Dzewaltski, Estabrooks and Johnston 2002) that may have an impact throughout life (Telama and Yang 2000). This is also a valuable time to promote physical activity as it has been found that physical activity declines as children reach adolescence (Booth et al. 2002; Salmon et al. 2004; Trost 2005). Adolescence represents a stage of life when interventions associated with physical activity are vital. It is important to determine potential barriers and motivators to physical activity participation.

\section{Culture}

There is very little published research on the effect of culture on physical activity in Australian children and adolescents (Wilson and Dollman 2007). Attitudes to physical activity vary within cultures including gender (Centre for Culture, Ethnicity and Health 2006), parental support, and importance in relation to other activities such as academic success (Wilson and Dollman 2007). Further investigation in this area could provide valuable insight into suitable culturally specific interventions.

\section{Socioeconomic status}

Research suggests a higher prevalence of obesity among low socioeconomic status children (Dollman and Pilgram 2005; Salmon et al. 2005); however, there are mixed theories on the impact of socioeconomic status and participation in physical activity by children. While some research indicates variation between socioeconomic status and physical activity participation (Salmon et al. 2005) other research shows no significant correlation between socioeconomic status and physical activity patterns in children and adolescents (Martin et al. 2005; Raudsepp 2006; Spinks et al. 2006). Research conducted by Spinks et al. (2006) found that children from low-income families were more likely to walk or cycle for transport. Salmon et al. (2005) also found that children attending schools in low socioeconomic areas were more likely to walk to school than those in high socioeconomic areas. Similar results were noted in a South Australian study (Harten and Olds 2004). The impact of socioeconomic status could vary between general physical activity and organised sport where ongoing costs are required. Age of children may also impact on socioeconomic restrictions. Informal physical activity is often appropriate for younger children whereas as they grow older preference may be directed to more organised activities that involve financial cost (Ferreira et al. 2006). Jury et al. (2006) found that children in South Australia living in higher socioeconomic areas engaged in more organised sport than those from lower socioeconomic areas. Potential barriers suggested by Boshoff et al. (2007) that are likely to have a greater impact on 
children of low socioeconomic status include: cost of organised sport, safety, restricted means of private transport and limited access to facilities (Boshoff et al. 2007; Chinn et al. 1999). In a recent South Australian survey Jury et al. (2006) also found a relationship between children spending more than two hours per day involved in screen-based activity and living in low socioeconomic areas. This is supported by subsequent research conducted by Dollman et al. (2007).

\section{Environmental settings}

Understanding environmental factors that facilitate or restrict physical activity for children and adolescents is vital in developing effective intervention strategies (Ferreira et al. 2006; Wilson and Dollman 2007). The family and home environment are instrumental in influencing physical activity: family structure, parental support and the home setting potentially encourage or inhibit physical activity (Salmon et al. 2004). The home environment is changing, particularly with regard to outdoor play spaces (Dollman, Norton and Norton 2005). Access to opportunities for physical activity such as having a yard (Brunton et al. 2005) and outdoor play equipment increases opportunities to be active (Ferreira et al. 2006; Spinks et al. 2006). As Dollman et al. (2007) argue, there is a need for further research to investigate physical and social characteristics of the home environment and its impact on physical activity in children.

Proximity of facilities and services, street design, housing density, availability of public transport, safety, traffic and accessibility to pedestrian and bicycle facilities all play a significant role in physical activity opportunities (Brunton et al. 2005; Centre for Disease Control and Prevention 2007; Salmon et al. 2004). Active transport levels in Australian children are low (Harten and Olds 2004) and this warrants further research (Bauman et al. 2002). Environmental factors such as safety and accessible pedestrian and bicycle facilities will support an increase in desirability of active transport (Harten and Olds 2004).

\section{School settings}

Schools provide an ideal opportunity to provide health education principles and support health-promoting interventions (Dollman and Lewis 2007; Dzewaltski et al. 2002) that can be ongoing through curriculum and complement a whole-school approach (Booth and Okely 2005). Schools are in a position to influence the attitudes and behaviour of a large proportion of the young population irrespective of socioeconomic status, gender, culture or disadvantage (Ferreira et al. 2006). Schoolbased sport and physical activity provides the most opportunity for children and adolescents to participate in physical activity (Salmon et al. 2004). Most children and adolescents attend school and schools are able to provide the facilities and infrastructure needed to promote physical activity (Booth and Okely 2005). Schools provide an ideal medium to provide knowledge and education. However, there are many demands on the time spent at school and not all teachers are adequately trained in teaching physical activity and health education. Education plays a vital role in intervention programs (Pyle et al. 2006). However, education alone will not increase physical activity (Egger et al. 1999). 
Cleland et al. (2008) investigated the role of compulsory physical activity within Australian schools and its association with physical activity levels, fitness and overweight. Findings indicate that despite enforced physical activity in schools, outside of the associated benefits of any physical activity, rates of overall physical activity and fitness were not significantly influenced in childhood or in the long term. This supports the Dollman and Lewis (2007) premise that schools alone cannot provide sustainable outcomes in increasing physical activity levels in children that will last into adulthood (Cleland et al. 2008). Without support outside of school hours any positive results are unlikely to be sustainable (Dollman and Lewis 2007).

\section{Summary}

Most Australian children and adolescents are physically active; however the percentage that is not warrants concern. There are many influences on participation in physical activity for children and adolescents ranging from parental influences, gender and age, culture, socioeconomic status, and social and physical environments.

\section{Interventions}

We have conducted literature reviews to gain a broad national and international perspective of physical activity interventions involving children and adolescents and characteristics that deem them effective. Here we summarise the outcomes of the discussed interventions. We cannot always identify the strengths, weaknesses and limitations of each intervention due to inconsistent research design and reporting methods. For the purpose of this paper we have taken into account any positive effects that can be further explored.

Several authors (Salmon et al. 2007; Sharma 2006; Timperio, Salmon and Ball 2004; Van Sluijs, McMinn and Griffin 2007) have conducted systematic literature reviews of physical activity interventions for children and adolescents. We have chosen these reviews because they provide a thorough representation of Australian and global interventions. The reviews have encompassed school-based and out-ofschool programs; a variety of settings; a diverse range of intervention designs including one or all of curriculum modification, environmental changes and policy strategies; varying size and age samples; and consider various socioeconomic positions. While most reviewed physical activity interventions, the aim of some interventions included lifestyle issues such as nutrition and sedentary behaviours, but as physical activity was measured independently they have been included. In total 163 interventions were reviewed; however, there was some overlap of interventions between authors. The majority of interventions targeted primary school children. As Sharma (2006) suggested, this is a time when behaviour can be influenced to affect future behaviours. However, with current trend data showing declines in physical activity as children become older there is a need to maintain interventions and to investigate further the barriers to physical activity in adolescence. Opportunities to participate in physical activity through increased facilities, and policy changes that influence environment, school curriculum, afterschool activities and create awareness will support increases in physical activity 
(Okely et al. 2008). A summary relating to interventions around physical activity targeting youth is in point form below.

\section{Key points from the literature}

- There is a paucity of research on physical activity trends and interventions in early childhood.

- Learned behaviours in early childhood potentially carry on throughout childhood; therefore this an ideal time to introduce sound habits and educate parents.

- Improving parental skills benefits the child and the parent.

- Multiple strategy interventions that incorporate education, support and repetition of the messages have proven effective.

- Curriculum-only strategies achieve minimal results as they do not take into account the 'bigger picture'.

- Strategies that combine curriculum, physical education classes and policy are highly effective.

- Family involvement is beneficial.

- The use of physical education specialists within schools attracts and maintains interest and enjoyment.

- Provision of extra-curricular physical activity programs and noncompetitive activities support school-based activities.

- Long-term strategies that use repeated messages encourage sustainability of results.

- Interventions are less effective in children of low socioeconomic populations, in many cases due to a lack of finances.

- Boys are more active than girls but participate in more screen-based activities. Therefore initiatives are needed to promote an increase in activity for girls and a decrease in screen-based activity for boys.

- $\quad$ Adolescence is a time when physical activity levels decline. Therefore this is an area that requires further investigation to determine barriers and potential motivators.

- Interventions need to encompass interpersonal, intrapersonal and environmental approaches and consider all socioeconomic variables. 
- School interventions should incorporate a whole-of-school approach including: curriculum, physical education sessions, policy and environmental strategies, and involve family.

- There is a need to develop a clear understanding of varying cultural impacts on physical activity in children.

- There is a need for further research to investigate physical and social characteristics of the home environment and its impact on physical activity in children.

- Further research is required within low socioeconomic and disadvantaged groups to identify specific barriers to physical activity participation.

\section{Conclusion and recommendations}

It is clear from the literature that there is a need for more research into physical activity interventions in early childhood, in particular for children aged zero to five. The research that does exist highlights the need for multifaceted approaches that educate, support and are repetitive in their strategy. Having parents involved in the intervention and enhancing the child-parent feedback loop is essential to enhance the success of physical activity interventions as well as community-based capacity building.

We have identified key areas in which future physical activity interventions need to be aimed and some of the major issues confronting youth in terms of successful physical activity interventions. The school environment provides an important site around which such interventions can take place. However, 'curriculum only' strategies do not appear to work. The need to combine curriculum and education approaches with good quality physical education teachers is paramount. Similarly the development of extra-curricula and non-curricula school-based physical activity initiatives provides consistent further exposure to physical activity. It is arguable that taking a whole-school approach to promoting physical activity is highly beneficial in endorsing and reinforcing positive physical activity messages that can be sustained over time. However, schools in lower socioeconomic areas have less effective results in physical activity interventions irrespective of whole-school approaches. It is imperative to include parents within physical activity interventions where possible to enhance the prospect of successful outcomes.

Further research must be undertaken in working with diverse population groups such as CALD and low socioeconomic groups as these will require their own specific set of interventions.

Recommendations for future strategies arising from the reviews include the need for:

- longer term interventions with follow-ups

- $\quad$ appropriately powered studies with adequate sample sizes 
- $\quad$ a specific focus on physical activity

- $\quad$ studies that target high-risk groups

- $\quad$ studies that are conducted in a variety of specific settings

- culturally specific and appropriate strategies to meet the needs of all socioeconomic groups

- $\quad$ strengthening of evidence bases through rigorous design

- $\quad$ encouraging children to be active in a variety of ways in and out of school hours.

\section{References}

Australian Bureau of Statistics 2006, Physical activity in Australia: a snapshot 2004-05, ABS, Canberra.

Bauman, AE, Bellew, B, Vita, P, Brown, W and Owen, N 2002, Getting Australia active: towards better practice for the promotion of physical activity, National Public Health Partnership, Melbourne.

Bauman, A, Ford, I and Armstrong, T 2001, Trends in population levels of reported physical activity in Australia, 1997, 1999 and 2000, Australian Sports Commission, Canberra.

Bauman, A, Owen, N and Leslie, E 2000, 'Physical activity and health outcomes: epidemiological evidence, national guidelines and public health initiatives', Australian Journal of Nutrition and Dietetics, vol. 57, no. 4, pp. 229-232.

Booth, ML and Okely, AD 2005, 'Promoting physical activity among children and adolescents: the strengths and limitations', Health Promotion Journal of Australia, vol. 16, no. 1, pp. 52-54.

Booth, ML, Okely, AD, Chey, T, Bauman, AE and Macaskill, P 2002, 'Epidemiology of physical activity participation among New South Wales school students', Australian and New Zealand Journal of Public Health, vol. 26, no. 4, pp. 371-374.

Boshoff, K, Dollman, J and Magary, A 2007, 'An investigation into the protective factors for overweight among low socio-economic status in children', Health Promotion Journal of Australia, vol. 18, no. 2, pp. 135-142.

Brunton, G, Thomas, J, Harden, A, Rees, R, Kavanagh, J, Oliver, S et al. 2005, 'Promoting physical activity amongst children outside of physical education 
classes: a systematic review integrating intervention studies and qualitative studies', Health Education Journal, vol. 64, pp. 323-338.

Burton, NW, Oldenburg, B, Sallis, JF and Turrell, G 2007, 'Measuring psychological, social, and environmental influences on leisure-time physical activity among adults', Australian and New Zealand Journal of Health Promotion, vol. 31, no. 1, pp. 36-43.

Campbell, KJ and Hesketh, KD 2007, 'Strategies which aim to positively impact on weight, physical activity, diet and sedentary behaviours in children from zero to five years: a systematic review of the literature', Obesity Reviews, vol. 8, pp. 327-338.

Centre for Culture, Ethnicity and Health 2006, Engaging culturally and linguistically diverse communities in physical activity: a discussion paper, Centre for Culture, Ethnicity and Health, Melbourne, www.ceh.org.au/resources/resbyceh.html (accessed 28 April 2008).

Centre for Disease Control and Prevention 2007, ACES: Active Community Environments Initiative, http://www.cdc.gov/nccdphp/dnpa/physical/health_professionals/active_envir onments/aces.htm (accessed 28 April 2008).

Chinn, DJ, White, M, Harland, J, Drinkwater, C and Raybould, S 1999, 'Barriers to physical activity and socio-economic position: Implications for health promotion', Journal of Epidemiology and Community Health, vol. 53, no. 3, pp. 191-193.

Cleland, V, Dwyer, T, Blizzard, L and Venn, A 2008, 'The provision of compulsory school physical activity: associations with physical activity, fitness and overweight in childhood and twenty years later', International Journal of Behavioral Nutrition and Physical Activity, vol. 5, no. 14, pp. 5-14.

Department of Health and Ageing 2004a, Australia's physical activity recommendations for 5-12 year olds, Commonwealth of Australia, Canberra.

Department of Health and Ageing 2004b, Australia's physical activity recommendations for 12-18 year olds, Commonwealth of Australia, Canberra.

Dollman, J and Lewis, F 2007, 'Trends in health attitudes and self-perceptions among school-age South Australians between 1985 and 2004', Australian and New Zealand Journal of Public Health, vol. 31, no. 5, pp. 407-413.

Dollman, J, Norton, K and Norton, L 2005 'Evidence for secular trends in children's physical activity behaviour', British Journal of Sports Medicine, vol. 39, pp. 892-897. 
Dollman, J and Pilgram, A 2005, 'Changes in body composition between 1997 and 2002 among South Australian children: influences of socio-economic status and location of residence', Australian \& New Zealand Journal of Public Health, vol. 29, no. 2, pp. 29-36.

Dollman, J, Ridley, K, Magarey, A, Martin, M and Hemphill, E 2007, 'Dietary intake, physical activity and TV viewing as mediators of the association of socioeconomic status with body composition: a cross-sectional analysis of Australian youth', International Journal of Obesity, vol. 31, pp. 45-52.

Drummond, M, Drummond, C, Dollman, J and Abery, E 2008, An investigation of physical activity among low socio-economic and disadvantaged groups, SA Health, Adelaide.

Dzewaltski, DA, Estabrooks, PA and Johnston, JA 2002, 'Healthy youth places promoting nutrition and physical activity', Health Education Research, vol. 17, no. 5, pp. 541-551.

Egger, G, Donovan, R, Swinburn, B, Giles-Corti, B and Bull, F 1999, Physical activity guidelines for Australians: scientific background report, Commonwealth Department of Health and Aged Care, Canberra.

Ferreira, I, van de Horst, K, Wendel-Vos, W, Kremers, S, van Lenthe, FJ and Brug, $\mathrm{J}$ 2006, 'Environmental correlates of physical activity in youth: a review and update’, Obesity Reviews, vol. 8, pp. 129-154.

Fogelholm, M, Nuutinen, O, Pasanen, E, Myohanen, E and Saatela, T 1999, 'Parent-child relationship of physical activity patterns and obesity', International Journal of Obesity, vol. 23, no. 12, pp. 1262-1268.

Harrison, M, Burns, CF, McGuiness, M, Heslin, J and Murphy, NM 2006, 'Influence of a health education intervention on physical activity and screen time in primary school children: "Switch Off-Get Active", Journal of Science and Medicine in Sport, vol. 9, pp. 388-394.

Harten, N and Olds, T 2004, 'Patterns of active transport in 11-12 year old Australian children', Australian and New Zealand Journal of Public Health, vol. 28, no. 2, pp. 167-172.

Jury, H, Cottrell, J, Gill, T and Taylor, A 2006, Report on children's activities, January 2004 - December 2006, South Australian Monitoring and Surveillance System (SAMSS), Population Research and Outcome Studies Unit, Adelaide.

Kahn, EB, Ramsey, LT, Brownson, RC, Heath, GW, Howze, EH, Powell, KE et al. 2002, 'The effectiveness of interventions to increase physical activity: a systematic review', American Journal of Preventive Medicine, vol. 22, no. 4, pp. 73-107. 
Martin, M, Dollman, J, Norton, K and Robertson, I 2005, 'A decrease in the association between the physical activity patterns of Australian parents and their children, 1985-1997', Journal of Science and Medicine in Sport, vol. 8, no. 1, pp. 71-76.

Mathers, C, Vos, T and Stevenson, C 1999, Burdon of disease and injury in Australia, AIHW Catalogue PHE 17, Australian Institute of Health and Welfare, Canberra.

Okely, AD, Booth, ML, Hardy, L, Dobbins, T and Denney-Wilson, E 2008, 'Changes in physical activity participation from 1985-2004 in a statewide survey of Australian adolescents', Archives of Pediatric Medicine, vol. 162, no. 2, pp. 176-180.

Pyle, A, Sharkey, J, Yetter, G, Felix, E, Fullang, M and Poston, WSC 2006, 'Fighting an epidemic: the role of schools in reducing childhood obesity', Psychology in the Schools, vol. 43, no. 3, pp. 361-367.

Raudsepp, L 2006, 'The relationship between socio-economic status, parental support and adolescent physical activity', Acta Paediatrica, vol. 95, pp. 9398.

Salmon, J, Booth, ML, Phongsavan, P, Murphy, NM and Timperio, A 2007, 'Promoting physical activity participation among children and adolescents', Epidemiologic Reviews, vol. 29, no. 1, pp. 144-159.

Salmon, J, Telford, A and Crawford, D 2004, The children's leisure activities summary report, Centre for Physical Activity and Health Research, Victoria.

Salmon, J, Timperio, A, Cleland, V and Venn, A 2005, 'Trend's in children's physical activity and weight status in high and low socio-economic status areas of Melbourne, Victoria, 1985-2001', Australian and New Zealand Journal of Public Health, vol. 29, no. 4, pp. 337-342.

Sharma, M 2006, 'International school-based interventions for preventing obesity in children’, Obesity Reviews, vol. 8, pp. 155-167.

Short, S 2004, 'Fat is a fairness issue', Griffith Review, no. 4, http://www.griffithreview.com/edition-4/170-policy/477.html (accessed 24 November 2010).

Spinks, A, Macpherson, A, Bain, C and McClure, R 2006, 'Determinants of sufficient daily activity in Australian primary school children', Journal of Paediatrics and Child Health, vol. 42, pp. 674-679.

Stephenson, J, Bauman, A, Armstrong, T, Smith, B and Bellow, B 2000, The costs of illness attributable to physical inactivity in Australia: a preliminary study, 
Commonwealth Department of Health and Aged Care and Australian Sports Commission, Canberra.

Telama, R and Yang, XL 2000, 'Decline of physical activity from youth to young adulthood in Finland', Medicine, Science and Sports Exercise, vol. 32, no. 9, pp. 1617-1622.

Timperio, A, Salmon, J and Ball, K 2004, 'Evidence-based strategies to promote physical activity among children, adolescents and young adults: review and update', Journal of Science and Medicine in Sport, vol. 7, no. 1 (suppl), pp. 20-29.

Trost, SG 2005, Discussion paper for the development of recommendations for children's and youths' participation in health promoting physical activity, Department of Health and Ageing, Canberra.

Van Sluijs, EMF, McMinn, AM and Griffin, SJ 2007, 'Effectiveness of interventions to promote physical activity in children and adolescents: systematic review of controlled trials', British Medical Journal, no. 335, pp. 703-707.

Wilson, AN and Dollman, J 2007 'Social influences on physical activity in Angloand Vietnamese-Australian adolescent males in a single sex school', Journal of Science and Medicine in Sport, vol. 10, pp. 147-155.

World Health Organization 2004, World Health Organization global strategy on diet, physical activity and health, World Health Organization, Geneva. 